FTUV-07-1607

\title{
Tau anomalous magnetic moment form factor at Super B/Flavor factories.
}

\author{
J. Bernabéu ${ }^{\text {a }, ~ G . ~ A . ~ G o n z a ́ l e z-S p r i n b e r g ~}{ }^{\text {b }}$ J. Papavassiliou ${ }^{\text {a }}$ \\ J. Vidal ${ }^{\text {a }}$ \\ ${ }^{a}$ Departament de Física Teòrica Universitat de València, E-46100 \\ Burjassot, València, Spain \\ and \\ IFIC, Centre Mixt Universitat de València-CSIC, València, Spain \\ ${ }^{\mathrm{b}}$ Instituto de Física, Facultad de Ciencias, Universidad de la República, Iguá 4225, \\ 11400 Montevideo, Uruguay
}

\begin{abstract}
The proposed high-luminosity B/Flavor factories offer new opportunities for the improved determination of the fundamental physical parameters of standard heavy leptons. Compared to the electron or the muon case, the magnetic properties of the $\tau$ lepton are largely unexplored. We show that the electromagnetic properties of the $\tau$, and in particular its magnetic form factor, may be measured competitively in these facilities, using unpolarized or polarized electron beams. Various observables of the $\tau$ 's produced on top of the $\Upsilon$ resonances, such as cross-section and normal polarization for unpolarized electrons or longitudinal and transverse asymmetries for polarized beams, can be combined in order to increase the sensitivity on the magnetic moment form factor. In the case of polarized electrons, we identify a special combination of transverse and longitudinal $\tau$ polarizations able to disentangle this anomalous magnetic form factor from both the charge form factor and the interference with the Z-mediating amplitude. For an integrated luminosity of $15 \times 10^{18} b^{-1}$ one could achieve a sensitivity of about $10^{-6}$, which is several orders of magnitude below any other existing high- or low-energy bound on the magnetic moment. Thus one may obtain a QED test of this fundamental quantity to a few $\%$ precision.
\end{abstract}

\section{Introduction}

Magnetic properties of elementary particles are of paramount importance both to theoretical and experimental high energy physics. The electron anomalous magnetic moment [1] is measured with the highest precision available in

Preprint submitted to Nuclear Physics B 24 October 2018 
physics nowadays:

$$
a_{e}=\mu_{e} / \mu_{B}-1=\frac{g_{e}-2}{2}==(1159.6521810 \pm 0.0000007) \times 10^{-6}
$$

and the best determination of the fine structure constant $\alpha$ is obtained from this electron property. The theoretical predictions in Quantum Electrodynamics (QED), obtained by Schwinger in 1948 [2], was the first one-loop computation in quantum field theories to be confronted with experiment:

$$
a_{e}=\frac{\alpha}{2 \pi}=\simeq 0.00116
$$

Actually, precision measurements of the anomalous magnetic moments do not only test QED (up to 4-loops), but the electroweak and hadronic interactions as well. In fact, since anomalous magnetic moments are chirality flipping quantities, weak quantum corrections to the muon anomalous magnetic moment are enhanced by a factor of the order of $m_{\mu}^{2} / m_{e}^{2} \simeq 40000$ compared to that of the electron; the measured value is [3]

$$
\begin{aligned}
a_{\mu} & =\mu_{\mu} /\left(e \hbar / 2 m_{\mu}\right)-1=\frac{g_{\mu}-2}{2} \\
& =(1165.92080 \pm 0.00054 \pm 0.00033) \times 10^{-6} .
\end{aligned}
$$

In addition, new physics models [4], and especially those furnishing massenhancements comparable to that of the Standard Model (SM), can be constrained from these extremely precise measurements, nowadays a subject of intense activity [5].

In comparison with these values, our experimental knowledge on the magnetic moment of the $\tau$ lepton is rather poor. While the electron and muon anomalous magnetic moments are known with more than seven figures, the PDG limit for the $\tau$ lepton magnetic moment anomaly is [1] :

$$
-0.052<a_{\tau}<0.013(95 \% \text { C.L. })
$$

These numbers are more than one order of magnitude bigger than the firstorder QED contribution, given in Eq. (2). Recent computations have reanalyzed both weak and hadronic corrections for the $\tau$ lepton magnetic anomaly within the SM, with an excellent agreement with previous computations [6]. The higher-order QED contributions are at the level of 1\% compared to the one-loop result of Schwinger, while hadronic and weak corrections are at the level of $0.001 \%$ and $0.04 \%$, respectively. 
The experimental determination of the anomalous magnetic moment of the fast-decaying $\tau$ is very different from that of the stable or relatively long-lived electron and muon, simply because one does not have the time to measure its interaction with an external electromagnetic field. Instead, the magnetic information is carried by the cross-section or partial widths for the $\tau$ pair production, together with spin matrices or angular distributions of the $\tau$ decay products. For example, the PDG bounds in Eq.(4) where obtained by the DELPHI Collaboration [7] from the LEP2 data for the total cross-section for the reaction $e^{+} e^{-} \rightarrow e^{+} e^{-} \tau^{+} \tau^{-}$, assuming that any deviation from the tree level SM prediction was exclusively due to magnetic anomaly [8].

The magnetic anomaly, together with analogous quantities related to the weak magnetism, i.e. the magnetic coupling with the $\mathrm{Z}$, have been already investigated experimentally $[7,9]$. The contributions to the magnetic and weak magnetic anomalies from physics beyond the SM have also been studied in the context of both low- and high-energy physics [10]. It is important to emphasize at this point that, strictly speaking, the magnetic moment anomaly is defined with all three fields entering into the interaction vertex on their massshell. However, in several of the aforementioned experiments the kinematics are such that the $\tau$ 's or the photon are in fact off-shell; therefore, what one actually measures is the corresponding form-factor (for some value of the momentum transfer) rather than $a_{\tau}$ itself. This is usually accomplished under the additional assumption that, of all possible form factors appearing in the off-shell vertex, the one corresponding to the magnetic moment gives the dominant contribution. Such experiments furnish bounds on the contributions to the magnetic moments from physics beyond the SM (since the scale of new physics is rather high, these latter contributions are practically "on-shell"). This is the point of view adopted in [10], where the most stringent modelindependent limit for the magnetic properties is obtained:

$$
-0.007<a_{\tau}^{\text {NewPhys. }}<0.005(95 \% \text { C.L. })
$$

The one-loop SM contribution to the magnetic form factor was computed in [11] long time ago, for arbitrary values of the photon "off-shellness" $\left(q^{2}\right)$ and with the charged fermions on-shell. The form factor obtained depends on the electroweak gauge-fixing parameter [12], and becomes gauge-independent only in the limit $q^{2} \rightarrow 0$. On the other hand, the pure QED corrections to the off-shell form factor are automatically gauge-independent, for any value of $q^{2}$.

LEP has been the main source of data on $\tau$-pair production until the advent of the $\mathrm{B}$ factories and their upgrades. In the near future they are expected to be superseded by several orders of magnitude, thanks to the high-luminosity Super B Factories, where $10^{11} \sim 10^{12} \tau$ pairs will be produced [13]. Motivated by these possibilities, CP-odd spin correlations have already been studied in [14], for low-energy physics. In addition, polarized beams are also being 
considered [13]; their implementation would allow the possibility of defining and measuring new observables, related to the linear $\tau$ polarization, not yet considered in the literature with respect to the magnetic properties.

In this paper we propose new observables in order to explore the poorly known magnetic properties of the $\tau$ lepton, using the highest statistics facilities available nowadays and in the near future. Cross-sections and asymmetries for resonant $\tau$ pair-production on top of the $\Upsilon$ resonances are studied. In particular we show that some of these observables, with the same discrete symmetry transformations as the magnetic moment, allow one to measure these properties with a precision up to $10^{-6}$. We compute the contribution of the magnetic form factor to the cross-section and the normal linear $\tau$ polarization -for unpolarized electron beams- and both transverse and longitudinal $\tau$ polarizations for polarized $e^{-}$-beams.

The fact that in this class of experiments one will be measuring the magnetic form-factor rather than the anomaly provides a unique opportunity to observe strong flavor-dependent effects, encoded in the momentum-dependence of the form factor, in the context of pure QED. Indeed, whereas the Schwinger correction (the leading order value of the magnetic form factor at $q^{2}=0$ ) is universal (i.e. independent of the fermion masses), the running of the form factors depends strongly on the mass of the fermion interacting with the photon. Thus, whereas the corresponding electron form factor practically vanishes at the values of $q^{2}$ that we consider ( $\Upsilon$ resonance), the $\tau$ form factor drops only to about one quarter of its initial value, because the heavy $\tau$ mass slows down the running considerably. In addition, for $q^{2}>4 m_{\tau}^{2}$ the form factor develops an imaginary part, which, as we will demonstrate, can also be experimentally measured.

The paper is organized as follows: In section (2) the magnetic form factors are defined, and their one-loop QED prediction (real and imaginary parts) reported. In section (3) the $\tau$ pair production at Super B factories is studied, and expressions for the differential cross-section and the normal asymmetry are derived. In section (4) polarized beams observables are considered, with particular emphasis on the transverse and longitudinal asymmetries. In section (5) the advantages of operating at the $\Upsilon$ resonances are discussed, and the role of the weak contributions, especially that of the $Z$ boson, considered. Finally, in section (6) we estimate the sensitivity expected for the anomalous magnetic moment form factor obtained from these observables, and present our conclusions and final remarks. 


\section{$2 f \bar{f} \gamma$ vertex form factors}

The most general Lorentz invariant structure describing the interaction of a vector boson $V$ with two on-shell fermions $f \bar{f}$ can be written in terms of six form factors:

$$
\begin{aligned}
\left\langle f\left(p_{-}\right) \bar{f}\left(p_{+}\right)\right| & J^{\mu}(0)|0\rangle=e \bar{u}\left(p_{-}\right)\left[\left(F_{1}+F_{4} \gamma_{5}\right) \gamma^{\mu}\right. \\
& \left.+\frac{1}{2 m_{f}}\left(i F_{2}+F_{3} \gamma_{5}\right) \sigma^{\mu \nu} q_{\nu}+\frac{1}{2 m_{f}}\left(i F_{5}+F_{6} \gamma_{5}\right) q^{\mu}\right] v\left(p_{+}\right)
\end{aligned}
$$

where $q=p_{+}+p_{-}$. Since the two fermions are on-shell the form factors $F_{i}$ appearing in Eq. (6) are functions of $q^{2}$ and $m_{f}^{2}$ only.

In addition, if the current $J^{\mu}$ is conserved, we must have

$$
i \frac{q^{2}}{2 m_{f}} F_{5}+\left(\frac{q^{2}}{2 m_{f}} F_{6}-2 m_{f} F_{4}\right) \gamma_{5}=0 \Rightarrow\left\{\begin{array}{l}
F_{5}=0 \\
F_{6}=\frac{4 m_{f}^{2}}{q^{2}} F_{4}
\end{array}\right.
$$

so that the final expression for the gauge invariant $f \bar{f} \gamma$ vertex reduces to:

$$
\begin{aligned}
& \left\langle f\left(p_{-}\right) \bar{f}\left(p_{+}\right)\left|J^{\mu}(0)\right| 0\right\rangle= \\
& \quad e \bar{u}\left(p_{-}\right)\left[\gamma^{\mu} F_{1}+\frac{1}{2 m_{f}}\left(i F_{2}+F_{3} \gamma_{5}\right) \sigma^{\mu \nu} q_{\nu}+\left(q^{2} \gamma^{\mu}-q^{\mu} \not q\right) \gamma_{5} F_{A}\right] v\left(p_{+}\right)(8)
\end{aligned}
$$

In this expression, $F_{1}$ parametrizes the vectorial part of the electromagnetic current $\left(F_{1}(0)=1\right), F_{A}=-F_{4} / q^{2}$ is the so-called anapole moment, while $F_{2}$ and $F_{3}$ parametrize the usual magnetic and electric dipole moments, respectively, i.e.

$$
F_{2}(0)=a_{f}, \quad d_{f}=\frac{e}{2 m_{f}} F_{3}(0) .
$$

The electric dipole moment is a $\mathrm{CP}$ violating magnitude, whose value vanishes in the SM up to three loops for leptons. Observables able to disentangle it from the rest have been studied in $[14,15]$ using techniques similar to those presented in the present paper; therefore, it is not going to be considered here. The P-odd anapole moment differs from zero due to weak corrections. In cross-sections its contribution will be suppressed by factors of $q^{2} / M_{Z}^{2}$ compared to the leading QED corrections, so that its determination will remain below the sensitivity of the proposed observables. As emphasized already in the 
Introduction, and as is clear from Eq. (9), $F_{2}\left(q^{2}\right)$ coincides with the anomaly $a_{\tau}$ only at $q^{2}=0$. In Super B factories the squared center-of-mass energy $s=q^{2} \approx(10 \mathrm{GeV})^{2}$, and therefore $F_{2}\left(q^{2}\right)$ is no longer the magnetic anomaly.

When attempting to extract the value of $F_{2}$ from scattering experiments (as opposed to using, say, a background magnetic field) one encounters additional complications due to the contributions of various other Feynman graphs, not related to the magnetic form factor. For example, in the case of $e^{+} e^{-} \longrightarrow \tau^{+} \tau^{-}$ that we will consider, one receives contributions not only from the usual $s$ channel one-loop vertex corrections but also from box diagrams. The contributions of the latter may interfere in the experimental determination of what we call $F_{2}\left(q^{2}\right)$, i.e. the magnetic part coming only from the vertex, and should be somehow "subtracted out". This may be done either by computing the box contributions and subtracting them from the cross-section, or by performing the measurement in a kinematic region where the boxes happen to be numerically subleading. The strategy we propose in this paper for eliminating the contamination from the boxes is to measure the observables on top of the $\Upsilon$ resonances; in this kinematic regime the (non-resonant) box diagrams are numerically negligible, and only one loop corrections to the $\gamma f \bar{f}$ vertex are relevant.

The direct text-book computation of the magnetic part of the standard oneloop QED vertex yields [16]

$$
F_{2}(s)=\left(\frac{\alpha}{2 \pi}\right) \frac{2 m_{\tau}^{2}}{s} \frac{1}{\beta}\left(\log \frac{1+\beta}{1-\beta}-i \pi\right), \quad \text { for } \quad q^{2}=s>4 m_{\tau}^{2}
$$

where $\alpha$ is the fine structure constant and $\beta=\left(1-4 m_{\tau}^{2} / s\right)^{1 / 2}$ is the velocity of the $\tau$. For $M_{\Upsilon} \sim 10 \mathrm{GeV}$,

$$
F_{2}\left(M_{\Upsilon}^{2}\right)=(2.65-2.45 i) \times 10^{-4}
$$

Evidently, at this energy the real and imaginary parts are of the same order.

Note that the above expression for $F_{2}(s)$ is gauge-independent, despite being an off-shell amplitude. This fact may be easily verified through an explicit calculation of the vertex diagram; more generally, the gauge-independence of $F_{2}(s)$ may be understood in terms of the way the gauge-cancellations organize themselves in the QED $S$-matrix elements. Specifically, the vacuum polarization is gauge-independent by itself; the gauge-dependence of the direct box cancels exactly against that of the crossed box; the gauge-dependence of the vertex correction can therefore cancel only against the fermion self-energy graphs renormalizing the external (on-shell) fermions. The latter however are proportional to $\gamma_{\mu}$. Therefore the contribution of the vertex proportional to 
$\sigma_{\mu \nu} q^{\nu}$ must be individually gauge-independent [17].

$3 e^{+} e^{-} \longrightarrow \tau^{+} \tau^{-}$at Super B Factories.

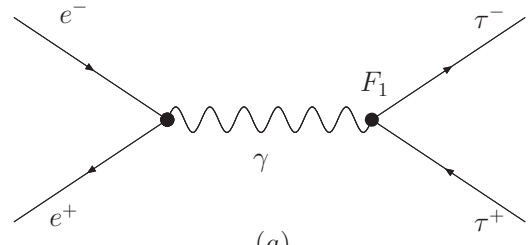

$(a)$

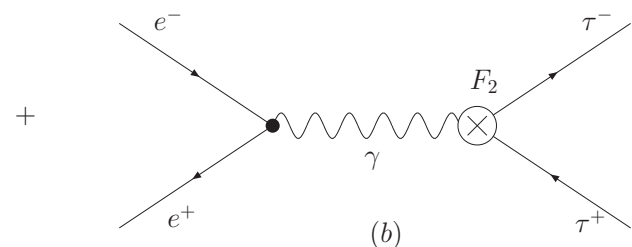

(b)

Fig. 1. Diagrams: (a) direct $\gamma$ exchange, (b) $F_{2}$ in $\gamma$ exchange.

In this section we first consider the $\tau$-pair production in $e^{+} e^{-}$collisions through direct $\gamma$ exchange (diagrams (a) and (b) in Fig. 1). Next, we will show that the basic results of this section still hold for resonant $\Upsilon$ production.

The spin-independent differential cross-section for $\tau$ pair-production can be written as:

$$
\frac{d \sigma^{0}}{d \cos \theta_{\tau^{-}}}=\frac{\pi \alpha^{2}}{8 s} \beta\left[\left(2-\beta^{2} \sin ^{2} \theta_{\tau^{-}}\right)\left|F_{1}(s)\right|^{2}+4 \operatorname{Re}\left\{F_{1}(s) F_{2}(s)^{*}\right\}\right]
$$

Note that, at one loop, we have the identity

$$
\operatorname{Re}\left\{F_{1}(s) F_{2}(s)^{*}\right\}=\operatorname{Re}\left\{F_{2}(s)\right\} \text {. }
$$

The $\theta_{\tau^{-}}$angle is determined in the center-of-mass $(\mathrm{CM})$ frame by the outgoing $\tau^{-}$and the incoming $e^{-}$momenta. As can be seen from Eq. (12), a precise measurement of the $\theta_{\tau^{-}}$angle allows to fit the differential cross-section and obtain a measurement for the $\operatorname{Re}\left\{F_{2}(s)\right\}$ form factor. In B/Flavor factories, this procedure requires that the $\tau$ production plane and direction of flight are fully reconstructed. In Ref. [18] it is shown that this can be achieved if both $\tau$ 's decay semileptonically. Following these ideas, the differential cross-section for $e^{+} e^{-} \longrightarrow \gamma \longrightarrow \tau^{+}\left(\vec{s}_{+}\right) \tau^{-}\left(\vec{s}_{-}\right) \longrightarrow h^{+} \bar{\nu}_{\tau} h^{-} \nu_{\tau}$ can then be written as [19]:

$$
\begin{aligned}
\frac{d \sigma}{d \cos \theta_{\tau^{-}}} & =\frac{\pi \alpha^{2}}{2 s} \beta\left[\left(2-\beta^{2} \sin ^{2} \theta_{\tau^{-}}\right)\left|F_{1}(s)\right|^{2}+4 \operatorname{Re}\left\{F_{2}(s)\right\}\right] \\
& \times \operatorname{Br}\left(\tau^{-} \rightarrow h^{-} \nu_{\tau}\right) \operatorname{Br}\left(\tau^{+} \rightarrow h^{+} \bar{\nu}_{\tau}\right)
\end{aligned}
$$

The real part of $F_{2}$ can be measured by a fit to Eq. (14), with a sensitivity that will be given basically by the statistical error, smeared by the precision in the determination of the angle of the outgoing $\tau$. Results presented in Table 1 
of Section 6 only consider statistical errors. The integrated cross-section from Eq. (14) will provide, to leading order in $\alpha\left(F_{1}=1, F_{2}=0\right)$, the normalization for all the asymmetries considered in this paper.

As can be seen from Eq. (14), the differential cross-section does not depend on the imaginary part of $F_{2}$, as expected. The imaginary part of $F_{2}$ is a T-odd, $\mathrm{C}$ - and P-even quantity; therefore, a suitable observable to look for its effects will be the normal (to the scattering plane) polarization of the outgoing $\tau$, as we will show in what follows.

\subsection{Normal Asymmetry}

In order to have sensitivity to the $\tau$ polarization, one has to measure the angular distribution of the decaying particles. In fact, in the cross-section all information on the imaginary part of $F_{2}$ is carried out by the linear spin-terms:

$$
\frac{d \sigma^{S}}{d \cos _{\tau^{-}}}=\frac{\pi \alpha^{2}}{4 s} \beta\left(s_{-}+s_{+}\right)_{y} Y_{+}
$$

where

$$
Y_{+}=\gamma \beta^{2}\left(\cos \theta_{\tau^{-}} \sin \theta_{\tau^{-}}\right) \operatorname{Im}\left\{F_{2}(s)\right\}
$$

and $\gamma=\sqrt{s} / 2 m_{\tau}$ is the dilation factor.

We work in the CM frame of reference, and the orientation of the coordinate system is the same as in Ref.[14]. The $\boldsymbol{s}_{ \pm}$vectors are the $\tau^{ \pm}$spin vectors in the $\tau^{ \pm}$rest system, $s_{ \pm}=\left(0, s_{ \pm}^{x}, s_{ \pm}^{y}, s_{ \pm}^{z}\right)$. Polarization along the directions $x, y, z$ correspond to what is called transverse, normal, and longitudinal polarizations, respectively. Eq.(16) shows that the contribution of the chirality flipping $F_{2}$ to the normal polarization is enhanced by a factor of $\gamma$ with respect to other possible non-chirality flipping contributions.

The polarization of the final fermion $\left(\tau^{ \pm}\right)$can be studied by looking at the angular distribution of its decay products. This again requires that only semileptonic $\tau$ decays must be considered [18]. The cross-section is [19]:

$$
\begin{aligned}
& d \sigma\left(e^{+} e^{-} \rightarrow \gamma \rightarrow \tau^{+}\left(\vec{s}_{+}\right) \tau^{-}\left(\vec{s}_{-}\right) \rightarrow h^{+} \bar{\nu}_{\tau} h^{-} \nu_{\tau}\right)=\frac{d \Omega_{h^{+}}}{4 \pi} \frac{d \Omega_{h^{-}}}{4 \pi} \\
& \times 4 d \sigma\left(e^{+} e^{-} \rightarrow \tau^{+}\left(\vec{n}_{+}^{*}\right) \tau^{-}\left(\vec{n}_{-}^{*}\right)\right) \operatorname{Br}\left(\tau^{+} \rightarrow h^{+} \bar{\nu}_{\tau}\right) \operatorname{Br}\left(\tau^{-} \rightarrow h^{-} \nu_{\tau}\right),
\end{aligned}
$$


with

$$
\vec{n}_{ \pm}^{*}=\mp \alpha_{ \pm} \frac{\vec{q}_{ \pm}^{*}}{\left|\vec{q}_{ \pm}^{*}\right|}=\mp \alpha_{ \pm}\left(\sin \theta_{ \pm}^{*} \cos \phi_{ \pm}, \sin \theta_{ \pm}^{*} \sin \phi_{ \pm}, \cos \theta_{ \pm}^{*}\right)
$$

The $\phi_{ \pm}$and $\theta_{ \pm}^{*}$ angles are the azimuthal and polar angles of the produced hadrons $h^{ \pm}\left(\hat{q}_{ \pm}^{*}\right)$ in the $\tau^{ \pm}$rest frame (the ${ }^{*}$ means that the quantity is given in the $\tau$ rest frame) and $\alpha_{h}$ is the polarization analyzer.

To preserve the normal polarization term in the cross-section of Eq. (17) one has to define a particular integration over the angular variables defined before. Indeed, the usual integration over the complete range of the $\tau^{-}$variables $d \Omega_{\tau^{-}}$ erases all the information on the $Y_{+}$term in the cross-section, so we must perform an asymmetric forward-backward (FB) integration on the $\theta_{\tau}$ angle. This can be done by defining

$$
\begin{aligned}
\sigma_{F B}\left(\vec{s}_{+}, \vec{s}_{-}\right) & \equiv 2 \pi\left\{\int_{0}^{1} d\left(\cos \theta_{\tau^{-}}\right)\left[\frac{d \sigma}{d \Omega_{\tau^{-}}}\right]-\int_{-1}^{0} d\left(\cos \theta_{\tau^{-}}\right)\left[\frac{d \sigma}{d \Omega_{\tau^{-}}}\right]\right\} \\
& =\frac{\pi \alpha^{2}}{6 s} \beta^{3} \gamma\left(s_{-}+s_{+}\right)_{y} \operatorname{Im}\left\{F_{2}(s)\right\},
\end{aligned}
$$

which retains the $\operatorname{Im}\left\{F_{2}\right\}$ term. Then, the cross-section in Eq. (17) can be written as

$$
\begin{aligned}
d^{4} \sigma_{F B}= & \frac{2 \pi \alpha^{2}}{3 s} \operatorname{Br}\left(\tau^{+} \rightarrow h^{+} \bar{\nu}_{\tau}\right) \operatorname{Br}\left(\tau^{-} \rightarrow h^{-} \nu_{\tau}\right) \frac{d \Omega_{h^{+}}}{4 \pi} \frac{d \Omega_{h^{-}}}{4 \pi} \\
& \times\left[\left(n_{-}^{*}\right)_{y}+\left(n_{+}^{*}\right)_{y}\right] \beta^{3} \gamma \operatorname{Im}\left\{F_{2}(s)\right\} .
\end{aligned}
$$

Integrating over as many kinematic variables as possible $\left(\theta_{ \pm}^{*}\right)$, without erasing the information on $F_{2}$, we finally find that the differential cross-section can be written as

$$
\begin{aligned}
\frac{d \sigma_{F B}}{d \phi_{ \pm}}= & \mp \frac{\pi \alpha^{2}}{12 s} \operatorname{Br}\left(\tau^{+} \rightarrow h^{+} \bar{\nu}_{\tau}\right) \operatorname{Br}\left(\tau^{-} \rightarrow h^{-} \nu_{\tau}\right) \\
& \times\left(\alpha_{ \pm}\right) \beta^{3} \gamma \operatorname{Im}\left\{F_{2}(s)\right\} \sin \phi_{ \pm} .
\end{aligned}
$$

To get an observable sensitive to the relevant signal, we must now define the azimuthal normal asymmetry as:

$$
A_{N}^{ \pm}=\frac{\sigma_{L}^{ \pm}-\sigma_{R}^{ \pm}}{\sigma}= \pm \alpha_{ \pm} \frac{1}{2\left(3-\beta^{2}\right)} \beta^{2} \gamma \operatorname{Im}\left\{F_{2}(s)\right\}
$$


where

$$
\sigma_{L}^{ \pm} \equiv \int_{\pi}^{2 \pi} d \phi_{ \pm}\left[\frac{d \sigma_{F B}}{d \phi_{ \pm}}\right], \quad \sigma_{R}^{ \pm} \equiv \int_{0}^{\pi} d \phi_{ \pm}\left[\frac{d \sigma_{F B}}{d \phi_{ \pm}}\right]=-\sigma_{L}^{ \pm}
$$

\section{Polarized $e^{-}$beams.}

For polarized electrons, available at Super B factories, an alternative procedure for measuring $F_{2}$ would be to consider the longitudinal and transverse polarizations of the outgoing $\tau$ 's.

Because $F_{1}$ and $\operatorname{Re}\left\{F_{2}\right\}$ have the same properties under $\mathrm{C}, \mathrm{P}$ and $\mathrm{T}$ symmetries, any single observable sensitive to one will also carry information on the other. Therefore, the extraction of the chirality flipping $\operatorname{Re}\left\{F_{2}\right\}$ requires two independent observables, where $F_{1}$ and $F_{2}$ enter with different coefficients; that would allow to express $F_{2}$ as a linear combination of the two measured observables. Following the notation and procedure of references $[10,15,20]$, our aim is to build observables that are linear in $F_{2}$.

$\operatorname{Re}\left\{F_{2}\right\}$ is even under $\mathrm{T}, \mathrm{C}$ and $\mathrm{P}$, while the longitudinal and transverse (to the scattering plane) polarizations of each $\tau$ are the only components of the spin matrix that are even under $\mathrm{T}$ and $\mathrm{C}$, but odd under $\mathrm{P}$. For this reason, an observable sensitive to $F_{2}$ will need an additional P-odd contribution coming, in our case, from longitudinally polarized electrons.

The linear spin-dependent part of the differential cross-section for $\tau$ pair production, with polarized electrons with helicity $\lambda$, can be written as

$$
\begin{aligned}
\left.\frac{d \sigma^{S}}{d \cos _{\tau^{-}}}\right|_{\lambda}=\frac{\pi \alpha^{2}}{8 s} \beta\{ & \left(s_{-}+s_{+}\right)_{y} Y_{+} \\
& \left.\quad+\lambda\left[\left(s_{-}+s_{+}\right)_{x} X_{+}+\left(s_{-}+s_{+}\right)_{z} Z_{+}\right]\right\},
\end{aligned}
$$

with

$$
\begin{aligned}
& X_{+}=\sin \theta_{\tau^{-}}\left[\left|F_{1}\right|^{2}+\left(2-\beta^{2}\right) \gamma^{2} \operatorname{Re}\left\{F_{2}\right\}\right] \frac{1}{\gamma} \\
& Z_{+}=\cos \theta_{\tau^{-}}\left[\left|F_{1}\right|^{2}+2 \operatorname{Re}\left\{F_{2}\right\}\right],
\end{aligned}
$$

and $Y_{+}$as defined in Eq. (16). Notice: i) The combination of the two form factors is different for the transverse and longitudinal $\tau$ polarization terms; ii) these two terms have different angular dependence. 
As can be seen from Eq.(25), Re $\left\{F_{2}\right\}$ contributes linearly to the longitudinal and transverse $\tau$ polarization. It may again be observed that, due to the fact that $F_{2}$ is a chirality-flip form factor, its contribution to the transverse polarization is enhanced by the factor $\gamma^{2}$ with respect to the chirality-non flipping factor $F_{1}$. This a very important fact for our purposes, because it will allow the accurate determination of $F_{2}$.

\subsection{Transverse Asymmetry}

Following a procedure similar to the one presented in section 3.1, it can be seen that the integration over the $\tau^{-}$variables $d \Omega_{\tau^{-}}$erases from Eq. (25) all information on the $Z_{+}$and $Y_{+}$terms. Then, the differential cross-section for the process $\left.e^{+} e^{-}\right|_{\text {Pol }} \rightarrow \gamma \rightarrow \tau^{+}\left(\vec{s}_{+}\right) \tau^{-}\left(\vec{s}_{-}\right) \rightarrow h^{+} \bar{\nu}_{\tau} h^{-} \nu_{\tau}$ is given by

$$
\begin{aligned}
\left.d^{4} \sigma^{S}\right|_{\lambda}= & \frac{\pi^{2} \alpha^{2} \beta}{2 s} \operatorname{Br}\left(\tau^{+} \rightarrow h^{+} \bar{\nu}_{\tau}\right) \operatorname{Br}\left(\tau^{-} \rightarrow h^{-} \nu_{\tau}\right) \frac{d \Omega_{h^{+}}}{4 \pi} \frac{d \Omega_{h^{-}}}{4 \pi} \\
& \times \beta \lambda\left[\left(n_{-}^{*}\right)_{x}+\left(n_{+}^{*}\right)_{x}\right] \frac{1}{\gamma}\left[\left|F_{1}\right|^{2}+\left(2-\beta^{2}\right) \gamma^{2} \operatorname{Re}\left\{F_{2}\right\}\right] .
\end{aligned}
$$

Subtracting the cross-sections for different helicities, ${ }^{1}$

$$
\left.\mathrm{d}^{2} \sigma^{S}\right|_{\mathrm{Pol}\left(e^{-}\right)} \equiv \frac{1}{2}\left[\left.d^{4} \sigma\right|_{\lambda=1}-\left.d^{4} \sigma\right|_{\lambda=-1}\right]
$$

and integrating over as many kinematic variables as possible, without erasing the information on the $F_{2}$ term, we get

$$
\begin{aligned}
\left.\frac{d \sigma^{S}}{d \phi_{ \pm}}\right|_{\operatorname{Pol}\left(e^{-}\right)}= & \mp \frac{\pi^{2} \alpha^{2} \beta}{16 s} \operatorname{Br}\left(\tau^{+} \rightarrow h^{+} \bar{\nu}_{\tau}\right) \operatorname{Br}\left(\tau^{-} \rightarrow h^{-} \nu_{\tau}\right) \\
& \times \frac{1}{\gamma}\left[\left|F_{1}\right|^{2}+\left(2-\beta^{2}\right) \gamma^{2} \operatorname{Re}\left\{F_{2}\right\}\right]\left[\left(\alpha_{ \pm}\right) \cos \phi_{ \pm}\right] .
\end{aligned}
$$

To get an observable sensitive to the relevant signal define the azimuthal transverse asymmetry as

$$
A_{T}^{ \pm}=\frac{\left.\sigma_{R}^{ \pm}\right|_{\mathrm{Pol}}-\left.\sigma_{L}^{ \pm}\right|_{\mathrm{Pol}}}{\sigma}
$$

$\overline{1}$ This subtraction eliminates also higher order absorptive parts that may be present. See Ref. [15] 


$$
=\mp \alpha_{ \pm} \frac{3 \pi}{8\left(3-\beta^{2}\right) \gamma}\left[\left|F_{1}\right|^{2}+\left(2-\beta^{2}\right) \gamma^{2} \operatorname{Re}\left\{F_{2}\right\}\right]
$$

where

$$
\begin{aligned}
\left.\sigma_{L}^{ \pm}\right|_{\mathrm{Pol}} \equiv \int_{\pi / 2}^{3 \pi / 2} d \phi_{ \pm}\left[\left.\frac{d \sigma^{S}}{d \phi_{ \pm}}\right|_{\operatorname{Pol}\left(e^{-}\right)}\right]= \pm \operatorname{Br}\left(\tau^{+} \rightarrow h^{+} \bar{\nu}_{\tau}\right) \operatorname{Br}\left(\tau^{-} \rightarrow h^{-} \nu_{\tau}\right) \\
\quad \times \alpha_{ \pm} \frac{(\pi \alpha)^{2} \beta}{8 s} \frac{1}{\gamma}\left[\left|F_{1}\right|^{2}+\left(2-\beta^{2}\right) \gamma^{2} \operatorname{Re}\left\{F_{2}\right\}\right] \\
\left.\sigma_{R}^{ \pm}\right|_{\mathrm{Pol}} \equiv \int_{-\pi / 2}^{\pi / 2} d \phi_{ \pm}\left[\left.\frac{d \sigma^{S}}{d \phi_{ \pm}}\right|_{\operatorname{Pol}\left(e^{-}\right)}\right]=-\left.\sigma_{L}^{ \pm}\right|_{\mathrm{Pol}}
\end{aligned}
$$

It is clear from Eq. (29) that in order to separate out $\operatorname{Re}\left\{F_{2}\right\}$, we need to remove the contribution of $F_{1}$. This can be done in two ways. The first is to use that $\left|F_{1}\right|^{2}=1$ at tree-level, and assume that any additional contribution to $F_{1}$ will be of the same order as $F_{2}$; since $F_{2}$ is a chirality flipping quantity, it will be enhanced by a factor $\gamma^{2}$ with respect to the additional $F_{1}$. Under these assumptions, a measurement of the $A_{T}$ asymmetry (subtracted with the tree level value $\left|F_{1}\right|^{2}=1$ ) will translate into a $\operatorname{Re}\left\{F_{2}\right\}$ measurement. The second way is to consider a new observable, relating $\left|F_{1}\right|^{2}$ and $\operatorname{Re}\left\{F_{2}\right\}$, and combine the two measurements to extract the value of $\operatorname{Re}\left\{F_{2}\right\}$. This can be done by defining a longitudinal asymmetry $\left(A_{L}\right)$ as follows.

\subsection{Longitudinal Asymmetry}

From Eqs. (24) and (25) it can be seen that, as happened with Eq. (19) for the normal asymmetry, an asymmetrical (FB) integration on the $\theta_{\tau}$ angle will select the longitudinal term of the cross-section

$$
\left.\sigma_{F B}^{S}\left(\vec{s}_{+}, \vec{s}_{-}\right)\right|_{\lambda} \equiv \frac{\pi \alpha^{2}}{4 s} \beta\left[\lambda\left(s_{-}+s_{+}\right)_{z} \widetilde{Z}_{+}+\frac{2}{3} \beta^{2} \gamma\left(s_{-}+s_{+}\right)_{y} \operatorname{Im}\left\{F_{2}\right\}\right]
$$

where $\widetilde{Z}_{+}=Z_{+} / \cos \theta_{\tau^{-}}$. Following a similar procedure as in the previous paragraph, and after subtracting for different helicities as was done in Eq. (27), but integrating over the azimuthal angles $\phi_{ \pm}$instead of the polar $\theta_{ \pm}^{*}$ ones, we end up with the final expression for the asymmetrical (FB) differential cross-section for polarized electrons: 


$$
\begin{aligned}
\left.\frac{d \sigma_{F B}^{S}}{d\left(\cos \theta_{ \pm}^{*}\right)}\right|_{\operatorname{Pol}\left(e^{-}\right)}= & \mp \frac{\pi \alpha^{2} \beta}{2 s} \operatorname{Br}\left(\tau^{+} \rightarrow h^{+} \bar{\nu}_{\tau}\right) \operatorname{Br}\left(\tau^{-} \rightarrow h^{-} \nu_{\tau}\right) \\
& \times\left[\left|F_{1}\right|^{2}+2 \operatorname{Re}\left\{F_{2}\right\}\right]\left[\left(\alpha_{ \pm}\right) \cos \theta_{ \pm}^{*}\right]
\end{aligned}
$$

Then, we define the longitudinal asymmetry as

$$
\begin{aligned}
& A_{L}^{ \pm}=\frac{\left.\sigma_{F B}^{ \pm}(+)\right|_{\mathrm{Pol}}-\left.\sigma_{F B}^{ \pm}(-)\right|_{\mathrm{Pol}}}{\sigma} \\
&=\mp \alpha_{ \pm} \frac{3}{4\left(3-\beta^{2}\right)}\left[\left|F_{1}\right|^{2}+2 \operatorname{Re}\left\{F_{2}\right\}\right],
\end{aligned}
$$

where

$$
\begin{aligned}
\left.\sigma_{F B}^{ \pm}(+)\right|_{\mathrm{Pol}} & \left.\equiv \int_{0}^{1} d\left(\cos \theta_{ \pm}^{*}\right) \frac{d \sigma_{F B}^{S}}{d\left(\cos \theta_{ \pm}^{*}\right)}\right|_{\mathrm{Pol}\left(e^{-}\right)}=\mp \alpha_{ \pm} \operatorname{Br}\left(\tau^{+} \rightarrow h^{+} \bar{\nu}_{\tau}\right) \\
& \times \operatorname{Br}\left(\tau^{-} \rightarrow h^{-} \nu_{\tau}\right) \frac{\pi \alpha^{2}}{4 s} \beta\left[\left|F_{1}\right|^{2}+2 \operatorname{Re}\left\{F_{2}\right\}\right] \\
\left.\sigma_{F B}^{ \pm}(-)\right|_{\mathrm{Pol}} & \left.\equiv \int_{-1}^{0} d\left(\cos \theta_{ \pm}^{*}\right) \frac{d \sigma_{F B}^{S}}{d\left(\cos \theta_{ \pm}^{*}\right)}\right|_{P o l\left(e^{-}\right)}=-\left.\sigma_{F B}^{ \pm}(+)\right|_{\mathrm{Pol}} .
\end{aligned}
$$

Combining Eq.(29) and Eq. (34) one can determine the real part of $F_{2}(s)$. Specifically,

$$
\operatorname{Re}\left\{F_{2}(s)\right\}=\mp \frac{8\left(3-\beta^{2}\right)}{3 \pi \gamma \beta^{2}} \frac{1}{\alpha_{ \pm}}\left(A_{T}^{ \pm}-\frac{\pi}{2 \gamma} A_{L}^{ \pm}\right) .
$$

\section{Observables on the $\Upsilon$ resonance}

As explained in the Introduction, our aim is to measure the observables on the top of the $\Upsilon$ peak where the $\tau$ pair-production is mediated by the resonance. The leading diagrams for the process $e^{+} e^{-} \rightarrow \Upsilon \rightarrow \tau^{+} \tau^{-}$are shown in Fig.2. Given that we are interested in $\tau$ pairs produced by the decays of the $\Upsilon$ resonances, we can use $\Upsilon(1 S)$ and $\Upsilon(2 S)$, since their decay rates into $\tau$ pairs have been measured and are sizeable. We assume that only the resonant diagrams (c) and (d) of Fig. 2 dominate the process on the $\Upsilon$ peaks, so no contribution from box diagrams has to be considered. As discussed in ref. [14], the $\tau$ pair-production at the $\Upsilon$ peak introduces the same $\tau$ polarization matrix terms as the direct production with a $\gamma$ exchange (diagrams (a) and (b), Fig. 1) that we have calculated in section 3. The only difference is an overall 


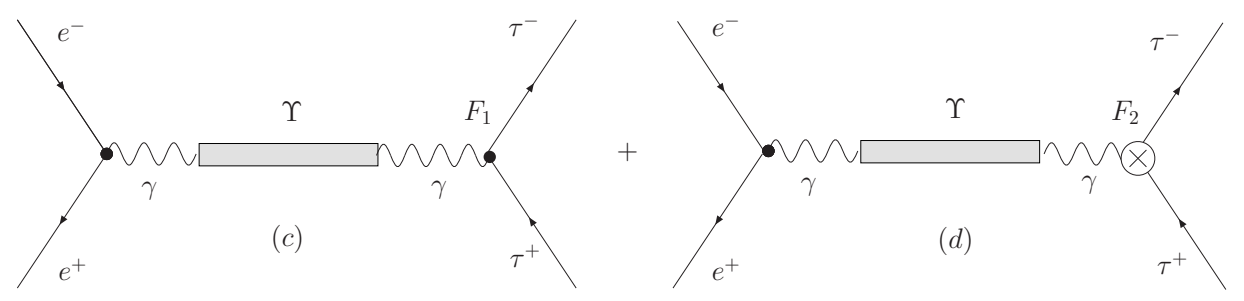

Fig. 2. Diagrams: (c) $\Upsilon$ production, (d) $F_{2}$ in $\Upsilon$ production

factor $|H(s)|^{2}$ which is responsible for the enhancement of the cross-section at resonant energies; the pure resonant amplitude is given by

$$
H\left(M_{\Upsilon}^{2}\right)=\frac{4 \pi \alpha Q_{b}^{2}}{M_{\Upsilon}^{2}} \frac{\left|F_{\Upsilon}\left(M_{\Upsilon}^{2}\right)\right|^{2}}{i \Gamma_{\Upsilon} M_{\Upsilon}}=-i \frac{3}{\alpha} B r\left(\Upsilon \rightarrow e^{+} e^{-}\right) .
$$

At the $\Upsilon$ peak, the interference of diagrams (a) and (d), plus the interference of diagrams (b) and (c), shown in Fig. 1 and 2, is exactly zero, and so is the interference of diagrams (a) and (c). Finally, the only contributions proportional to the $F_{2}$ come from the interference of diagrams (c) and (d), while diagram (c) squared gives the leading contribution to the cross-section.

\section{$5.1 Z$ contribution on the $\Upsilon$ resonance.}

It is important to notice that the observables previously defined receive contributions also from the standard $Z-\gamma$ interference, computed at the resonance. The process has been studied in detail in ref. [21]; the dominant (resonant) contribution will come from the interference of diagram (c) in Fig.2 with diagrams (e) and (f) in Fig.3.
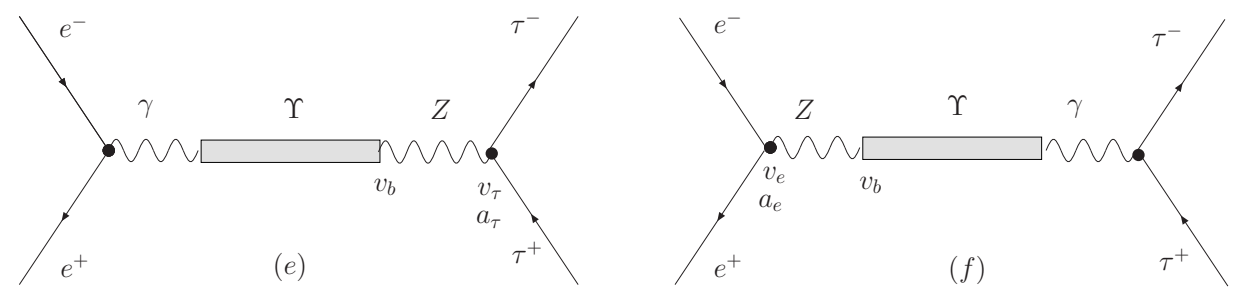

Fig. 3. $\gamma$ and $Z$ interchange on $\Upsilon$ production

Following a procedure similar to that explained in detail in [14], one can find the following relations among the amplitudes of diagrams (c), (e) and (f) with the non-resonant $\gamma$-mediated diagram (a) and $Z$-mediated diagram (g) of Fig. 4 .

$$
T_{c}=T_{a} \times H\left(M_{\Upsilon}^{2}\right), \quad T_{d}=T_{b} \times H\left(M_{\Upsilon}^{2}\right),
$$




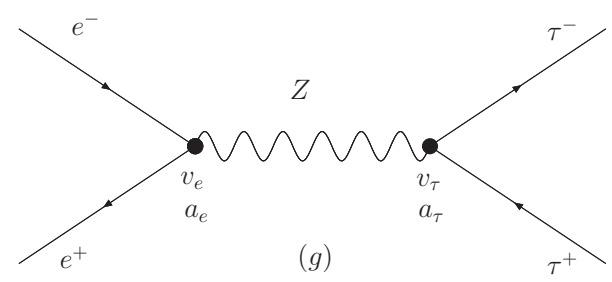

Fig. 4. Non-resonant $Z$ interchange

$$
\begin{aligned}
& T_{e}=T_{g}\left(a_{e} \rightarrow 0, v_{e} \rightarrow v_{b}\right) \times\left(H\left(M_{\Upsilon}^{2}\right) \frac{Q_{e}}{Q_{b}}\right), \\
& T_{f}=T_{g}\left(a_{\tau} \rightarrow 0, v_{\tau} \rightarrow v_{b}\right) \times\left(H\left(M_{\Upsilon}^{2}\right) \frac{Q_{e}}{Q_{b}}\right) .
\end{aligned}
$$

From these relations, the resonant $\gamma-\Upsilon-Z$ interference can be extracted from the purely $\gamma-Z$ interference. This was studied in Ref.[15] and the additional contributions to the spin-averaged cross-section and to its spin- dependent part are

$$
\begin{aligned}
\left.\frac{d \sigma^{0}}{d \Omega_{\tau^{-}}}\right|_{\lambda} ^{Z} & =-\frac{\alpha^{2} \beta}{8\left(2 s_{w} c_{w}\right)^{2}}\left|H\left(M_{\Upsilon}^{2}\right)\right|^{2} \frac{Q_{e}}{Q_{b}} v_{b}\left|P_{Z}\left(M_{\Upsilon}^{2}\right)\right|^{2} \\
\left.\frac{d \sigma^{S}}{d \Omega_{\tau^{-}}}\right|_{\lambda} ^{Z} & \left.=\frac{\alpha^{2} \beta}{8\left(2 s_{w} c_{w}\right)^{2}} \mid H\left(M_{\Upsilon}^{2}-M_{Z}^{2}\right) M_{0}^{2}\right) \\
& \times\left\{\Gamma_{Z} M_{Z}\left(s_{-}+s_{+}\right)_{y} Y_{+}^{Z}\right. \\
& +\left(M_{\Upsilon}^{2}-M_{Z}^{2}\right)\left[\left(s_{-}+s_{+}\right)_{x} X_{+}^{Z}+\left(\left.P_{Z}\left(M_{\Upsilon}^{2}\right)\right|^{2},\right.\right.
\end{aligned}
$$

where

$$
\begin{aligned}
& \left|P_{Z}\left(M_{\Upsilon}^{2}\right)\right|^{2}=\left[\left(M_{\Upsilon}^{2}-M_{Z}^{2}\right)^{2}+\Gamma_{Z}^{2} M_{Z}^{2}\right]^{-1}, \\
& a=-\frac{1}{2}, \quad v=-\frac{1}{2}+2 s_{w}^{2} \quad v_{b}=-\frac{1}{2}+\frac{2}{3} s_{w}^{2}, \quad Q_{b}=\frac{-1}{3}, \quad Q_{e}=-1, \\
& M_{0}^{Z}=\lambda a\left(2 \beta \cos \theta_{\tau^{-}}+2-\beta^{2} \sin ^{2} \theta_{\tau^{-}}\right)-2 v\left(2-\beta^{2} \sin ^{2} \theta_{\tau^{-}}\right) \\
& X_{+}^{Z}=\frac{1}{\gamma}\left[4 \lambda v-a\left(2+\beta \cos \theta_{\tau^{-}}\right)\right] \sin \theta_{\tau^{-}}, \\
& Z_{+}^{Z}=4 \lambda v \cos \theta_{\tau^{-}}-a\left[\beta\left(1+\cos ^{2} \theta_{\tau^{-}}\right)+2 \cos \theta_{\tau^{-}}\right] \\
& Y_{+}^{Z}=-\frac{\lambda}{\gamma} \beta a \sin \theta_{\tau^{-}} .
\end{aligned}
$$

To obtain the contribution of the $Z$-interference to both the cross-section and 
the normal asymmetry for unpolarized electrons, one must average over $\lambda$ helicities in Eqs.(40) and (41). Then,

$$
\begin{aligned}
\left.\frac{d \sigma^{0}}{d \Omega_{\tau^{-}}}\right|^{Z}= & \frac{\alpha^{2} \beta}{16 M_{\Upsilon}^{2}}\left|H\left(M_{\Upsilon}^{2}\right)\right|^{2}\left(2-\beta^{2} \sin ^{2} \theta_{\tau^{-}}\right) \\
& \times \underbrace{\frac{4 v}{\left(2 s_{w} c_{w}\right)^{2}} \frac{Q_{e} v_{b}}{Q_{b}} M_{\Upsilon}^{2}\left(M_{\Upsilon}^{2}-M_{Z}^{2}\right)\left|P_{Z}\left(M_{\Upsilon}^{2}\right)\right|^{2}}_{\zeta} \\
\left.\frac{d \sigma^{S}}{d \Omega_{\tau^{-}}}\right|^{Z}= & -\frac{\alpha^{2} \beta a}{8\left(2 s_{w} c_{w}\right)^{2}}\left(\left|H\left(M_{\Upsilon}^{2}\right)\right|^{2} \frac{Q_{e}}{Q_{b}} v_{b}\right)\left|P_{Z}\left(M_{\Upsilon}^{2}\right)\right|^{2}\left(M_{\Upsilon}^{2}-M_{Z}^{2}\right) \\
& \times\left\{\left(s_{-}+s_{+}\right)_{x} \frac{1}{\gamma}\left(2+\beta \cos \theta_{\tau^{-}}\right)\right. \\
& \left.+\left(s_{-}+s_{+}\right)_{z}\left[\beta\left(1+\cos ^{2} \theta_{\tau^{-}}\right)+2 \cos \theta_{\tau^{-}}\right]\right\} .
\end{aligned}
$$

Eq. (43) shows that the $Z$ contribution will only enter in the determination of $F_{1}$, with the suppression factor $\zeta \approx-1.576 \times 10^{-3}$. Furthermore, it is controlled by the small vector neutral coupling $v$ to leptons. This implies that the angular distribution is like that for $\left|F_{1}\right|^{2}$ and the measurement of $\operatorname{Re}\left\{F_{2}\right\}$ from the cross-section is not modified.

Similarly, Eq. (44) shows that there is no contribution from the $Z$-interference to the normal asymmetry, and the extraction of the $\operatorname{Im}\left\{F_{2}\right\}$ value from this observable through Eq. (22) is again not modified.

For polarized electrons, subtracting the cross-sections for different helicities and integrating, as was done in the previous section for the transverse asymmetry, one obtains the new contribution from Z-interference to Eqs. (30) and (31) as

$$
\begin{aligned}
\left.Z_{\sigma_{L}}^{ \pm}\right|_{\mathrm{Pol}} & =\frac{2 \pi \alpha^{2} \beta}{\left(2 s_{w} c_{w}\right)^{2}}\left|P_{Z}\left(M_{\Upsilon}^{2}\right)\right|^{2} \operatorname{Br}\left(\tau^{+} \rightarrow h^{+} \bar{\nu}_{\tau}\right) \operatorname{Br}\left(\tau^{-} \rightarrow h^{-} \nu_{\tau}\right) \\
& \times\left(M_{\Upsilon}^{2}-M_{Z}^{2}\right)\left|H\left(M_{\Upsilon}^{2}\right)\right|^{2} \frac{Q_{e}}{Q_{b}} v_{b}\left[\left(\frac{\beta^{2}}{3}-1\right) a \pm \frac{\pi v}{4 \gamma} \alpha_{ \pm}\right] \\
\left.Z_{\sigma_{R}}^{ \pm}\right|_{\mathrm{Pol}} & =\frac{2 \pi \alpha^{2} \beta}{\left(2 s_{w} c_{w}\right)^{2}}\left|P_{Z}\left(M_{\Upsilon}^{2}\right)\right|^{2} \operatorname{Br}\left(\tau^{+} \rightarrow h^{+} \bar{\nu}_{\tau}\right) \operatorname{Br}\left(\tau^{-} \rightarrow h^{-} \nu_{\tau}\right) \\
& \times\left(M_{\Upsilon}^{2}-M_{Z}^{2}\right)\left|H\left(M_{\Upsilon}^{2}\right)\right|^{2} \frac{Q_{e}}{Q_{b}} v_{b}\left[\left(\frac{\beta^{2}}{3}-1\right) a \mp \frac{\pi v}{4 \gamma} \alpha_{ \pm}\right]
\end{aligned}
$$


so that the azimuthal transverse asymmetry, $A_{T}^{ \pm}$of Eq. (29), reads

$$
Z_{A_{T}^{ \pm}}= \pm \alpha_{ \pm} \frac{3 \pi\left(2-\beta^{2}\right) \gamma}{8\left(3-\beta^{2}\right)} \overbrace{\left(\frac{4 v_{b} v M_{\Upsilon}^{2}\left(M_{\Upsilon}^{2}-M_{Z}^{2}\right)}{\gamma^{2}\left(2 s_{w} c_{w}\right)^{2}\left(2-\beta^{2}\right) Q_{b}}\left|P\left(M_{\Upsilon}^{2}\right)\right|^{2}\right)}^{\varepsilon}
$$

Similarly, the $Z$ contribution to the asymmetric cross-sections of (35) and (36) for the longitudinal polarization of $\tau$ 's with polarized electrons is

$$
\begin{aligned}
\left.{ }_{\sigma_{F B}^{ \pm}}^{ \pm}(+)\right|_{\mathrm{Pol}} & =-\frac{\pi \alpha^{2} \beta}{\left(2 s_{w} c_{w}\right)^{2}}\left|P_{Z}\left(M_{\Upsilon}^{2}\right)\right|^{2} \operatorname{Br}\left(\tau^{+} \rightarrow h^{+} \bar{\nu}_{\tau}\right) \operatorname{Br}\left(\tau^{-} \rightarrow h^{-} \nu_{\tau}\right) \\
& \times\left(M_{\Upsilon}^{2}-M_{Z}^{2}\right)\left|H\left(M_{\Upsilon}^{2}\right)\right|^{2} \frac{Q}{Q_{b}} v_{b}\left[\beta a \pm v \alpha_{ \pm}\right] \\
\left.{ }^{Z} \sigma_{F B}^{ \pm}(-)\right|_{\mathrm{Pol}} & =-\frac{\pi \alpha^{2} \beta}{\left(2 s_{w} c_{w}\right)^{2}}\left|P_{Z}\left(M_{\Upsilon}^{2}\right)\right|^{2} \operatorname{Br}\left(\tau^{+} \rightarrow h^{+} \bar{\nu}_{\tau}\right) \operatorname{Br}\left(\tau^{-} \rightarrow h^{-} \nu_{\tau}\right) \\
& \times\left(M_{\Upsilon}^{2}-M_{Z}^{2}\right)\left|H\left(M_{\Upsilon}^{2}\right)\right|^{2} \frac{Q}{Q_{b}} v_{b}\left[\beta a \mp v \alpha_{ \pm}\right]
\end{aligned}
$$

and the corresponding contribution to the longitudinal asymmetry, $A_{L}^{ \pm}$of Eq. (34), is given by

$$
Z_{L}^{ \pm}= \pm \alpha_{ \pm} \frac{3}{4\left(3-\beta^{2}\right)} \overbrace{\left(\frac{4 v_{b} v M_{\Upsilon}^{2}\left(M_{\Upsilon}^{2}-M_{Z}^{2}\right)}{\left(2 s_{w} c_{w}\right)^{2} Q_{b}}\left|P\left(M_{\Upsilon}^{2}\right)\right|^{2}\right)}^{\varepsilon^{\prime}} .
$$

At $\Upsilon$ energies, the values of the $\varepsilon$ and $\varepsilon^{\prime}$ factors are of the order $3.17 \times 10^{-4}$ and $3.15 \times 10^{-3}$, respectively. Contrary to what happens with the non-resonant contribution (which is two orders of magnitude smaller) this $\Upsilon$-mediated $\gamma-Z$ contribution must be taken into account when extracting $F_{2}$ from longitudinal and transverse asymmetries for polarized electrons. Nevertheless, as the $\gamma-$ $\Upsilon-Z$ interference proceeds through the vector neutral current coupling to leptons, the structure of this amplitude is like the one for the contribution of the charge form factor $F_{1}$. As a consequence, the same combination (37) of the two asymmetries $\left(A_{T}-\frac{\pi}{2 \gamma} A_{L}\right)$ able to cancel the contribution of $\left|F_{1}\right|^{2}$ automatically cancels the contribution of the $\mathrm{Z}$ interference too. We have thus shown that $\operatorname{Re}\left\{F_{2}\right\}$ can be separated out from other contributions without any ambiguities. 
Table 1

Sensitivity of the $F_{2}$ measurement at the $\Upsilon$ energy $\left(a b=\right.$ attobarn $\left.=10^{-18} b\right)$

\begin{tabular}{|c|c|c|c|}
\hline \multirow{3}{*}{ EXPERIMENT } & \multicolumn{3}{|c|}{ O B S E R V A B L E } \\
\hline & Cross Section & $\begin{array}{c}\text { Normal } \\
\text { Asymmetry }\end{array}$ & $\begin{array}{c}\text { Transverse and } \\
\text { Longitudinal } \\
\text { Asymmetry } \\
\text { combined }^{*}\end{array}$ \\
\hline & $\operatorname{Re}\left\{F_{2}\right\}$ & $\operatorname{Im}\left\{F_{2}\right\}$ & $\operatorname{Re}\left\{F_{2}\right\}$ \\
\hline $\begin{array}{c}\text { Babar+Belle } \\
2 a b^{-1}\end{array}$ & $4.6 \times 10^{-6}$ & $2.1 \times 10^{-5}$ & $1.0 \times 10^{-5}$ \\
\hline $\begin{array}{c}\text { Super B/Flavor Factory } \\
\text { (1 yr. running) } \\
15 a b^{-1}\end{array}$ & $1.7 \times 10^{-6}$ & $7.8 \times 10^{-6}$ & $3.7 \times 10^{-6}$ \\
\hline $\begin{array}{c}\text { Super B/Flavor Factory } \\
\text { (5 yrs. running) } \\
15 a b^{-1}\end{array}$ & $7.5 \times 10^{-7}$ & $3.5 \times 10^{-6}$ & $1.7 \times 10^{-6}$ \\
\hline
\end{tabular}

* Polarized electrons required

\section{Precision of the $F_{2}\left(M_{\Upsilon}^{2}\right)$ measurement and conclusions}

We can now estimate the precision that can be achieved on the determination of $F_{2}$ using the observables defined before. For our numerical analysis we assume a set of integrated luminosities for high statistics $B /$ Flavor factories. We also consider the $\pi^{ \pm} \overline{\nu_{\tau}}$ or $\rho^{ \pm} \overline{\nu_{\tau}}$ (i.e. $h_{1}, h_{2}=\pi, \rho$ ) decay channels for the traced $\tau^{ \pm}$, while we sum up over $\pi^{\mp} \nu_{\tau}$ and $\rho^{\mp} \nu_{\tau}$ hadronic decay channels for the non traced $\tau^{\mp}$.

In Table 1 , we show the sensitivity that can be achieved for the magnetic moment form factor $F_{2}$ in different scenarios: Babar + Belle at $2 a b^{-1}$, B/Flavor factory, 1 yr. running $\left(15 a b^{-1}\right)$ and 5 yrs. running $\left(75 a b^{-1}\right)$. The results presented in Table 1 only consider statistical errors. Almost all the defined observables show similar accuracy in the determination of $F_{2}$, but only the normal asymmetry is sensitive to its imaginary part. Sensitivities coming from the cross-section require an accurate determination of the $\theta_{\tau^{-}}$angle of the outgoing $\tau$ for a large variety of angles.

To summarize, we have shown that low energy data makes possible a determination of the $\tau$ lepton QED effects on the measurement of the $\tau$ anomalous magnetic form factor $F_{2}$ at the $\Upsilon$ energies. A fit to the cross-section and a measurement of the normal polarization of the outgoing $\tau$ will determine the real and the imaginary part of $F_{2}$ up to a precision of $10^{-6}$. Compared with 
the QED prediction of Eq. (11), we see that a positive signal appears and it can be tested to the percent level. The $\gamma-\Upsilon-Z$ interference will not affect the determination of $F_{2}$. Polarized electron beams also open the possibility to determine the value of $\operatorname{Re}\left\{F_{2}\right\}$ by looking at the transverse and longitudinal polarizations of a single $\tau$. The precision is again of the order $10^{-6}$. We have identified the precise combination of the transverse and longitudinal $\tau$ polarizations for polarized electrons which is able to disentangle the anomalous magnetic moment form factor from the contributions of both the charge form factor and, at the same time, the resonant $\gamma-\Upsilon-Z$ interference.

We conclude that the measurement of these sets of observables at the upcoming Super B factories should furnish a high accuracy determination of the rather poorly known magnetic properties of the $\tau$ lepton.

\section{Acknowledgements}

This work has been supported by CONICYT-PDT-54/94-Uruguay, by MEC and FEDER, under the grants FPA2005-00711 and FPA2005-01678, and by Generalitat Valenciana under the grant ACOMP07-093. The work of J.P. is financed by the Fundación General UV. J. B. acknowledges CERN Theory Unit for hospitality. J.V. also thanks Dr. J. Salt (IFIC) for helpful conversations.

\section{References}

[1] W.-M. Yao et al., Journal of Physics G 33, (2006) 1.

[2] J. Schwinger, Phys. Rev. 73 (1948) 416, Phys. Rev. 76 (1949) 790.

[3] G. W. Bennett et al. [Muon g-2 Collaboration], Phys. Rev. Lett. 89 (2002) 101804, Erratum- Phys. Rev. Lett. 89 (2002) 129903.

[4] T. Kinoshita and W. J. Marciano, in Quantum Electrodynamics, edited by T. Kinoshita (World Scientific, Singapore, 1990), pp. 419478.

[5] M. Passera, Nucl. Phys. Proc. Suppl. 162 (2006) 242; A. Czarnecki and W. J. Marciano, Phys. Rev. D 64 (2001) 013014.

[6] M. A. Samuel, G. w. Li and R. Mendel, Phys. Rev. Lett. 67 (1991) 668, Erratum- Phys. Rev. Lett. 69 (1992) 995; S. Eidelman and M. Passera, Mod. Phys. Lett. A 22 (2007) 159.

[7] J. Abdallah et al. [DELPHI Collaboration], Eur. Phys. J. C 35 (2004) 159.

[8] F. Cornet and J. I. Illana, Phys. Rev. D 53 (1996) 1181. 
[9] A. Heister et al. [ALEPH Collaboration], Eur. Phys. J. C 30 (2003) 291.

[10] G.A. González-Sprinberg, A. Santamaria, J. Vidal, Nucl. Phys. B 582 (2000) 3 .

[11] K. Fujikawa, B. W. Lee and A. I. Sanda, Phys. Rev. D 6 (1972) 2923.

[12] Gauge-independent off-shell form factors in non-Abelian gauge theories can in fact be defined, by resorting to the pinch technique, see, for example J. Papavassiliou, Phys. Rev. D 41 (1990) 3179; J. Papavassiliou and C. Parrinello, Phys. Rev. D 50 (1994) 3059; J. Bernabéu, J. Papavassiliou and J. Vidal, Phys. Rev. Lett. 89 (2002) 101802 [Erratum-ibid. 89 (2002) 229902].

[13] SuperB Conceptual Design Report, http:www.pi.infn.it/SuperB .

[14] J. Bernabéu, G. A. González-Sprinberg and J. Vidal, Nucl. Phys. B 701 (2004) 87.

[15] J. Bernabéu, G.A. González-Sprinberg and J. Vidal, Nucl. Phys. B 763 (2007) 283.

[16] C. Itzykson and J. B. Zuber, "Quantum Field Theory," New York, Usa: Mcgrawhill (1980) 705 P.(International Series In Pure and Applied Physics)

[17] This simple organization of the gauge cancellations becomes much more intricate in the case of non-abelian contributions; nonetheless, a gaugeindependent of-shell $F_{2}(s)$ can still be defined, see [12].

[18] J. H. Kuhn, Phys. Lett. B 313 (1993) 458.

[19] Y.S. Tsai Phys. Rev. D 4 (1971) 2821.

[20] J. Bernabéu, G.A. González-Sprinberg, M. Tung and J. Vidal, Nucl. Phys. B 436 (1995) 474.

[21] J. Bernabéu and P. Pascual, Phys. Lett. B 87 (1979) 69; Nucl. Phys. B 172 (1980) 93. 